\title{
A concise study on the expression of GFP protein in E. coli strain DH5a
}

\author{
Maryada Garg ${ }^{1}$ - Anoop K. Dobriyal ${ }^{2}$ \\ ${ }^{1}$ Department of Biotechnology, Pt.Jawahar lal Nehru Govt. College, Faridabad, Haryana \\ 2 Department of Zoology, HNB Garhwal University Campus, Pauri Garhwal-246001, Uttarakhand \\ *Corresponding author email: maryada.goyal@gmail.com
}

Received: 12.4.2021; Revised: 12.11.2021; Accepted: 16.11.2021

CSociety for Himalayan Action Research and Development

\begin{abstract}
The GFP protein is a protein of high interest for molecular biologists and biotechnologists. Since 1994, this protein has proved to be an indispensable tool for molecular biology and biotechnology research work. This protein requires only oxygen and an energy source like glucose to work. It gives a green colour in presence of UV to blue light. This protein can be attached with the foreign gene to track its expression. The main aim of the task is to introduce GFP into the given bacterial culture. Induction of the protein gene is followed by Bradford assay; quantification of protein is done using this. The results of gene induction are checked via SDSPAGE and western blot. All preparations are of standard grade and all readings are taken in triplicates. A standard graph is also made to find out the protein in the unknown.
\end{abstract}

Keywords: GFP, SDS-PAGE, foreign gene, induction, Bradford assay, quantification of protein.

\section{Introduction}

Millions of proteins are synthesized in the living cells, every second. Many of these proteins can be exploited in real life for various research and medical purposes. Many genes like alpha-amylase, blood protein genes, insulin, hormone genes, etc. have been expressed in other living cells; once introduced, these are known as recombinant genes. The proteins produced by these genes are called recombinant proteins. Once the gene is successfully introduced in the foreign cell, it is necessary to identify which cells have taken up the protein, and which not. Genes used for the analysis and identification of the recombinant protein are called as reporter genes.

The GFP (Green Fluorescent Protein) gene is one of the best examples of reporter genes used in gene expression. This protein appears green in the presence of ultraviolet or blue light. It is added to the foreign cell along with the gene of interest. The cells which have taken up the foreign DNA/gene, glow in ultraviolet or blue light. The green color is an indicator of gene expression. Note that initially GFP was isolated from jellyfish (Bio-Rad Explorer transformation kit).After gene expression, an analysis of the introduced protein is required. The analysis helps to identify how much protein expression will occur in a foreign cell. This is important for quantification purposes.

Since 1994, GFP has proved to be an indispensable tool for molecular biology and biotechnology research work. This protein requires only oxygen and an energy source like glucose to work. The 'mec' gene expression in $C$. elegans has been widely studied with the help of GFP protein. Various biochemical pathways of the animal and plant body have been studied using this protein. Various host-pathogen interactions have also been studied using GFP (Valdivia, et.al., 1996). 
The insulin protein has been widely expressed in bacterial cells. This was done to produce large quantities of the protein. This protein has immense importance in diabetes treatment.

\section{Materials and methods:}

E. coli strain $\mathrm{DH} 5 \alpha$ harboring the plasmid pGFP taken. This culture has been grown in Luria Broth (LB) containing ampicillin. The culture does not contain IPTG (Iso-propyl thio-galactoside). IPTG looks like allolactose. It can trigger lac operon. The experiment was conducted in 3 days. On day 1, GFP protein was expressed in E. coli strain $\mathrm{DH} 5 \alpha$. Ampicillin was taken as the marker, and the bacterial culture was grown in LB (Luria Bertini). IPTG was added to the culture.

The protein present in the sample was estimated using the Bradford Assay. To produce color, dye binding reagent was added. Readings were taken at $595 \mathrm{~nm}$. First, the

\section{Table 1: Results of the Bradford Assay}

\section{RESULT: Bradford assay}

The samples were loaded in 96 well plate as per template.

\begin{tabular}{|c|c|c|c|c|c|c|c|c|c|c|c|c|}
\hline & $\mathbf{1}$ & $\mathbf{2}$ & $\mathbf{3}$ & $\mathbf{4}$ & $\mathbf{5}$ & $\mathbf{6}$ & $\mathbf{7}$ & $\mathbf{8}$ & $\mathbf{9}$ & $\mathbf{1 0}$ & $\mathbf{1 1}$ & $\mathbf{1 2}$ \\
\hline A & 0.344 & 0.26 & 0.304 & 0.299 & 0.297 & 0.319 & 0.262 & 0.344 & 0.226 & 0.303 & 0.255 & 0.385 \\
\hline B & 0.3 & 0.3 & 0.412 & 0.48 & 0.641 & 0.634 & 0.958 & 0.908 & 1.283 & 1.307 & 1.748 & 1.636 \\
\hline C & 0.773 & 0.735 & 0.867 & 0.854 & 1.038 & 1.164 & 1.506 & 1.472 & & & & \\
\hline D & & & & & & & & & & & & \\
\hline E & & & & & & & & & & & & \\
\hline F & & & & & & & & & & & & \\
\hline G & & & & & & & & & & & & \\
\hline H & & & & & & & & & & & & \\
\hline
\end{tabular}

Using these readings, the average of the blank was calculated (Row 'A'). All samples were in duplicates. So, an average of the two samples was taken. Then this average was subtracted from the average of the blank to calculate the standard graph was prepared, using BSA (Bovine serum albumin) as standard. Two concentrations of BSA were taken; $1 \mu \mathrm{g} / \mu \mathrm{l}$ and $0.2 \mu \mathrm{g} / \mu \mathrm{l}$. Using the standard graph, the values of the protein present in the unknown were calculated. There were four unknown samples: original stock culture of bacteria (E. coli strain DH5 $\alpha$ ) in which IPTG had not been added, culture at 30 minutes, culture at 1 hour, and the culture at 2 hours.

Later, the samples were made to run on SDSPAGE (Sodium dodecyl sulfate/Polyacrylamide Gel Electrophoresis). Since there were four samples, so, four lanes were run against the ladder. GFP was located. Later, a western blot was conducted to confirm the results of the SDS-PAGE gel.

\section{Results:}

The results of the Bradford assay are given in Table 1.
Row $\mathrm{A}=$ blanks/background control Row B = standards (in duplicate)

Row $\mathrm{C}=$ samples (in duplicate) 
Although, only a few readings come under the equation is also given. straight line of the standard graph. The linear

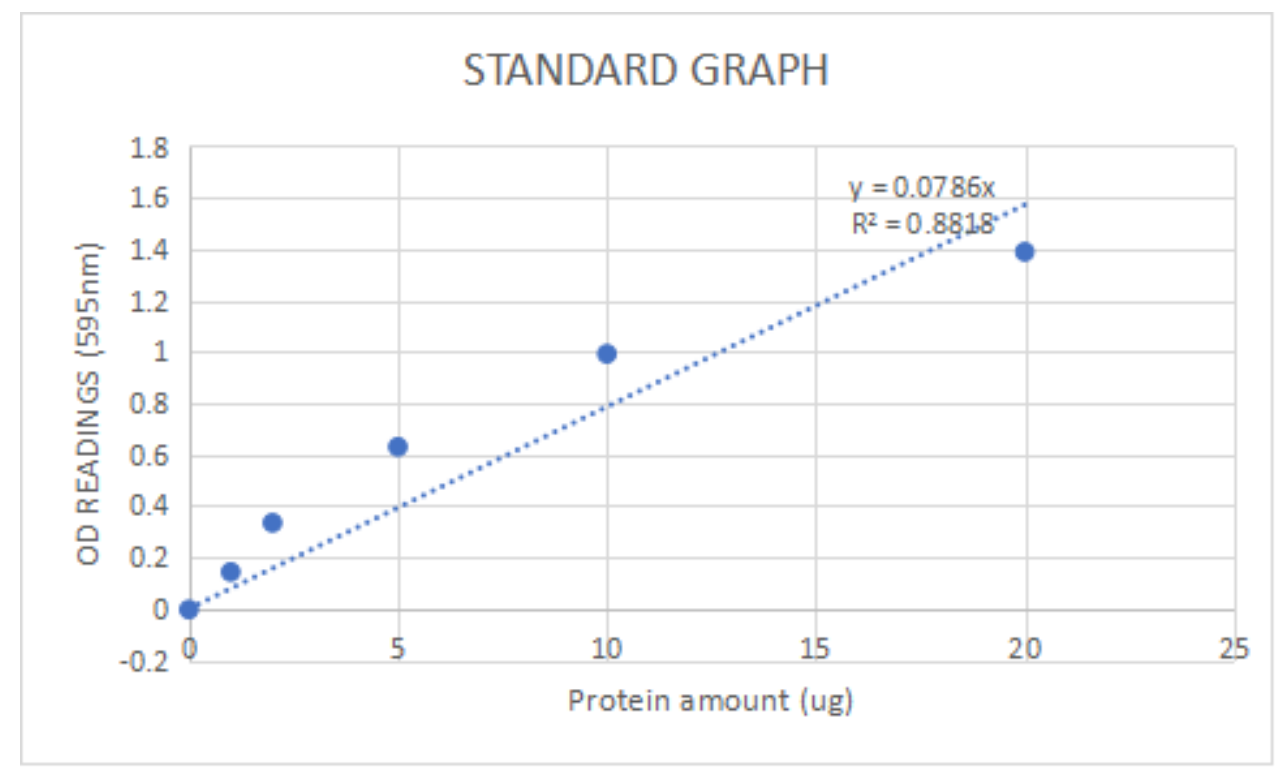

Figure 1: Standard curve along with the equation.

The linear equation and $\mathrm{R}^{2}$ values obtained was as follows:

$y=0.0786 x ;$ So, $x=y / 0.0786$

$\mathrm{R}^{2}=0.8818$

All readings were taken at $595 \mathrm{~nm}$.

Using this standard graph, the protein concentration of the unknown lysates were determined using the linear equation. Again, the normalized absorbance of the unknown samples (Row 'C') was the values of the Yaxis. These were plotted in the abovementioned linear equation to find the value of $\mathrm{x}$.

The corresponding values on the $\mathrm{X}$-axis were the concentrations of the protein present in the unknown samples. A detailed overview of the same is given in the Table 2

Table 2: Concentration of Protein in the unknown samples.

\begin{tabular}{lll}
\hline SAMPLE & $\begin{array}{l}\text { Protein amount }(\boldsymbol{\mu g}) \\
\mathbf{x = y} / \mathbf{0 . 0 7 8 6}\end{array}$ & $\begin{array}{l}\text { Protein concentration }(\boldsymbol{\mu g} / \boldsymbol{\mu l}) \\
=\mathbf{x} / \mathbf{2 0}\end{array}$ \\
\hline $\mathrm{T} 0$ & 5.735793 & 0.28679 \\
$\mathrm{~T}(0.5$ hour $)$ & 7.090755 & 0.354538 \\
$\mathrm{~T}(1$ hour $)$ & 10.15055 & 0.507528 \\
$\mathrm{~T}(2$ hour $)$ & 15.08694 & 0.754347
\end{tabular}

The resulting samples were subjected to gel electrophoresis (SDS-PAGE). All protein samples were denatured using SDS. The proteins, including GFP were denatured. This protein remains fluorescent even after denaturation. Coomassie stain was used for the visualization of the gel (Fig 2) 

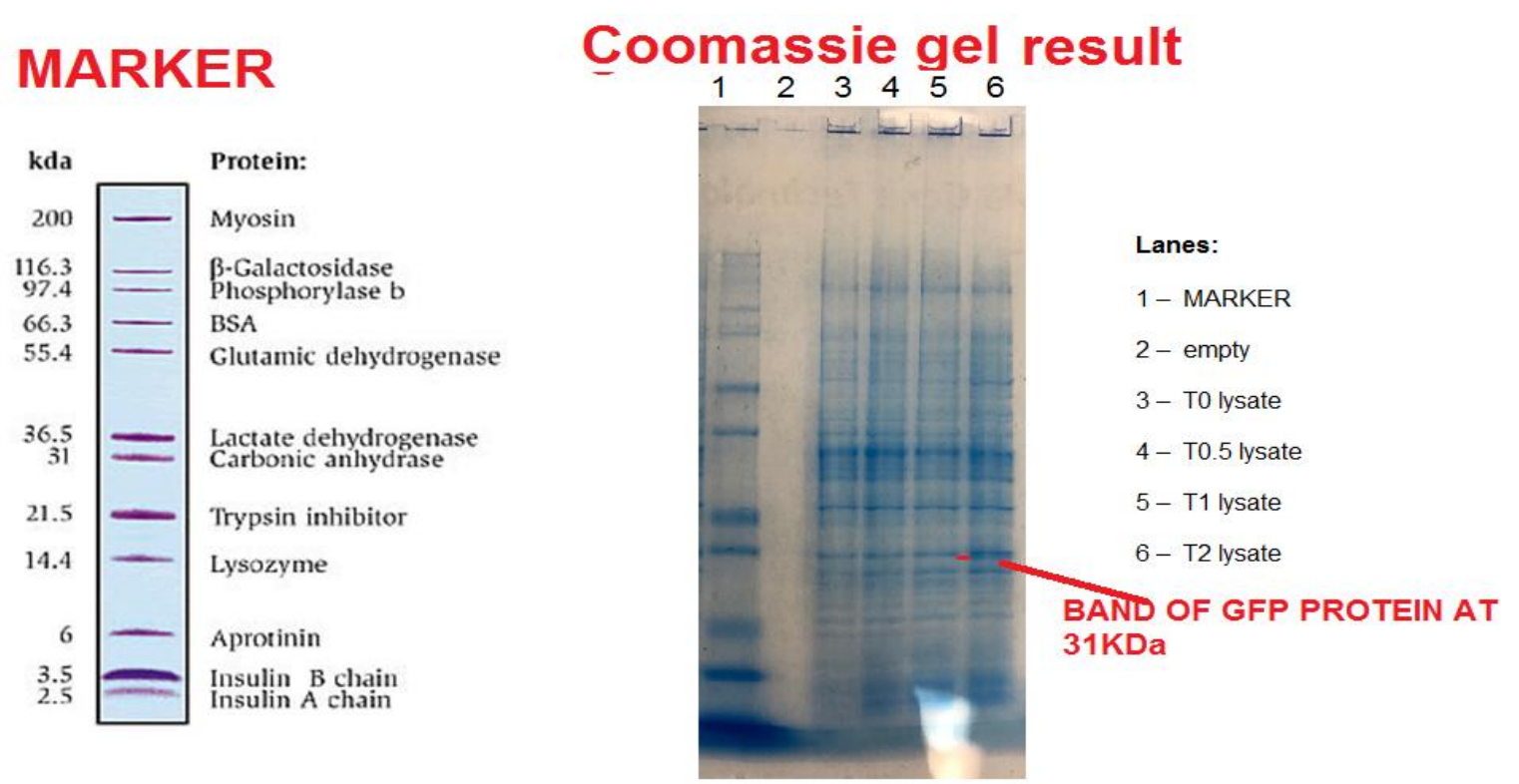

Figure 2: Coomassie gel electrophoresis. This gel is showing the separation of GFP from a mixture of denatured SDS proteins.

Later, a western blot analysis was done. In this, done. This was done to identify the presence specific probing of the lysate samples were of GFP (Fig 3)

\section{MARKER}

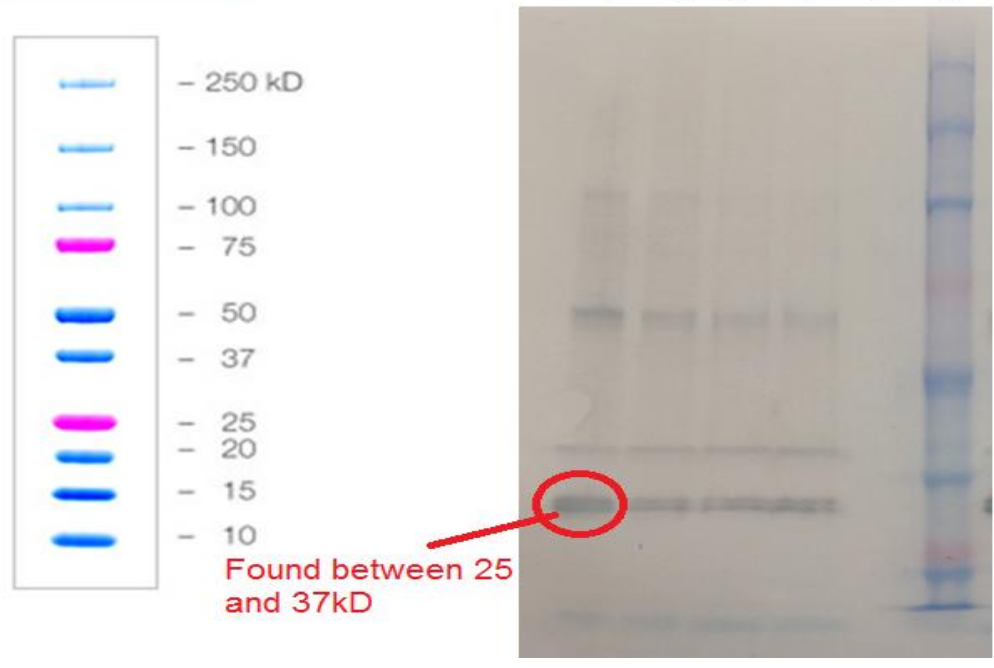

Lanes:

1 - T2 lysate

2 - T1 lysate

3 - T0.5 lysate

4 - To lysate

5 - empty

6 - MARKER

Figure 3: Western blot to detect GFP protein analysis.

It was seen that the GFP protein band becomes clearer and thicker with an increase in time. in T0 (lane 4 of western blot) the GFP band is lighter. On the other hand, a brighter and much clearer band can be seen in T2 (lane 1). The band of lane 1 is thicker than the others.

The sample lysate bands have been compared using the standard marker. The marker has 
been loaded in lane 6 of western blot; but, in the Coomassie gel it was loaded in lane 1 . The band appears at between 25 and 37kDa.

\section{Discussion}

About protein expression levels: Protein expression levels were increasing constantly from T0 to T2. The GFP band also became clearer. This means that GFP had integrated into the bacterial genome $E$. coli DH5 $\alpha$ initially. At T0, IPTG was not there. This means that a little bit of protein expression was there before the addition of IPTG also. After adding IPTG, the expression of the proteins increased. As time increased, protein expression kept increasing. The final sample was recorded after 2 hours. The overexpression of proteins was visible; because the size of the protein bands increased. A large and more conspicuous band of GFP at 2 hours indicates that gene expression was occurring constantly. The central dogma of biology can be explained and learned using this (Bio-Rad Explorer Transformation kit).

\section{Comparison between SDS-PAGE and}

Western blot: The SDS-PAGE explains about the cellular proteins. It gives a rough understanding of the proteins that are present in the four samples (T0 to T2). In T0, the stain is of less intensity, while in $\mathrm{T} 2$ the intensity of the stain is the highest. Individual bands in the gel did not change. All four lanes had almost the same number of bands. But the intensity kept on changing, proving that more protein is being produced as time lapses.

Every protein has its tertiary structure.
Cysteine residues are present between the protein chains; these contribute to the tertiary structure. If these residues are not removed, then the protein will not be linear. Such a protein cannot be run in the gel.

GFP was the fusion protein. When SDS is added to the sample, then, all proteins are denatured. But GFP is only partially denatured. To completely denature GFP, heat is required. The partially denatured protein remains fluorescent in the PAGE gel.

The cell lysate contains many proteins. A single band may contain many proteins. So, green color cannot be much visible in the SDS-PAGE gel. Because the green color of GFP blends with the blue color of the protein.

On the other hand, the western blot shows the green-colored bands (refer to the results section). The actual size of the lac $\mathrm{Z}$ protein can be detected using the western blot.

\section{Conclusion}

The most important part of the work was the qualitative and quantitative assessment of the proteins present in $\mathrm{T} 0$ to $\mathrm{T} 2$. The size of the proteins is also important. We have already noted the size of GFP (i.e. $31 \mathrm{kDa}$ ). The standards of the western blot are not very conspicuous, so, the exact size of proteins cannot be determined using the same. To find out the size of the proteins, PAGE gel was required (O'Connor, C.M., 2019).

In simple words, the gel results are necessary to find out the size of the protein samples, while the western blot is required to find out the GFP and its size. Proteins were detected on the western blot. These were present in all four 
samples.

In the first phase of the experiment, the GFP was introduced into the bacteria. The colonies of $E$. coli are qualitatively examined for green color. The presence of fluorescent green color means that the gene has been expressed. Between 25 to $37 \mathrm{kDa}$, the band of GFP was obtained. Later, from the gel results it was calculated that GFP protein is found at $31 \mathrm{kDa}$. This means that the size of the GFP protein is in this range. Other proteins were also detected in the samples. These include BSA, phosphorylase $b$, and $\beta$-galactosidase. The aim of the experiment was fully achieved.

Three important biotechnology techniques: transformation, electrophoresis, and western blotting were used. The versatility and robustness of the GFP protein were illustrated. The GFP protein is isolated from a jellyfish, named Aequorea victoria. GFP retains its fluorescent properties when cloned and expressed in E. coli; further, when isolated from $E$. coli and separated on polyacrylamide gels, then also GFP is visible. These amazing properties of GFP, and the powerful method of protein electrophoresis and western blotting allows visualizing the GFP protein band.

\section{References}

Bio-Rad Explorer Protein Electrophoresis of GFP: A pGLO bacterial transformation kit extension. http://www.biorad.com/webroot/web /pdf/lse/literature/M1660023.pdf Kumar A and Dharam Pal (2016) "Green fluorescent Protein and their applications in Advance Research 12 ", Research Journal of Applied Science, 01(01): 42-46.

O’Connor, C.M. (2019) "Micro-report 5: SDSPAGE and western blot analysis"

Biology Libre Texts, Boston

University. https://bio.libretexts. org/ $\underline{\text { Bookshelves /Cell_and_Molecular }}$ Biology Book\%3A_Investi ations in_Molecular_Cell_Biology_ (O'Connor)/Write_It_Up!/6\%3A Micro-report 5\% 3A SDS-PAGE and_western_blot_analysis

Valdivia RH, Hromockyj Alexander E, Monack Denise, Ramakrishnan Lalita and Falkow Stanley (1996) Applications for green fluorescent protein (GFP) in the study of host-pathogen interactions. Gene, 173 (1) :47-52. 